\title{
Forced ILW-Burgers Equation as a Model for Rossby Solitary Waves Generated by Topography in Finite Depth Fluids
}

\author{
Hongwei Yang, 1, 2, 3,4 Baoshu Yin, 1,4 \\ Yunlong Shi, ${ }^{2}$ and Qingbiao Wang ${ }^{5}$ \\ ${ }^{1}$ Institute of Oceanology, China Academy of Sciences, Qingdao 266071, China \\ ${ }^{2}$ Information School, Shandong University of Science and Technology, Qingdao 266590, China \\ ${ }^{3}$ Graduate School, Chinese Academy of Sciences, Beijing 100049, China \\ ${ }^{4}$ Key Laboratory of Ocean Circulation and Wave, Chinese Academy of Sciences, Qingdao 266071, China \\ ${ }^{5}$ Department of Resource and Civil Engineering, Shandong University of Science and Technology, \\ Taian 271019, China
}

Correspondence should be addressed to Baoshu Yin, baoshuyin@126.com

Received 19 July 2012; Accepted 6 September 2012

Academic Editor: Turgut Öziş

Copyright (C) 2012 Hongwei Yang et al. This is an open access article distributed under the Creative Commons Attribution License, which permits unrestricted use, distribution, and reproduction in any medium, provided the original work is properly cited.

The paper presents an investigation of the generation, evolution of Rossby solitary waves generated by topography in finite depth fluids. The forced ILW- (Intermediate Long Waves-) Burgers equation as a model governing the amplitude of solitary waves is first derived and shown to reduce to the KdV- (Korteweg-de Vries-) Burgers equation in shallow fluids and BO- (BenjaminOno-) Burgers equation in deep fluids. By analysis and calculation, the perturbation solution and some conservation relations of the ILW-Burgers equation are obtained. Finally, with the help of pseudospectral method, the numerical solutions of the forced ILW-Burgers equation are given. The results demonstrate that the detuning parameter $\alpha$ holds important implications for the generation of the solitary waves. By comparing with the solitary waves governed by ILW-Burgers equation and BO-Burgers equation, we can conclude that the solitary waves generated by topography in finite depth fluids are different from that in deep fluids.

\section{Introduction}

Solitary waves are finite-amplitude waves of permanent form which own their existence to a balance between nonlinear wave-steepening processes and linear wave dispersion. Typically, they consist of a single isolated wave, whose speed is an increasing function of the amplitude. The theoretical description of nonlinear solitary waves that have emerged in the mathematics 
and physics has received a great deal of attention in the last decades [1]. In atmospheric and oceanic circulation dynamics, Long [2] and Benney [3] first studied the barotropic Rossby waves employing horizontal shear velocity and obtained the conclusion that the amplitude of nonlinear solitary waves satisfied the KdV equation. The research on baroclinic Rossby waves was carried out by Redekopp in [4], the modified KdV (MKdV) equation as a model governing the amplitude of solitary waves was derived. Furthermore, Yamagata, Grimshaw, Boyd, and so on also discussed the solitary waves by virtue of the KdV equation [5-7]. Yano and Tsujimura classified the KdV-type Rossby solitary waves which were governed by the $\mathrm{KdV}$ and $\mathrm{MKdV}$ equations, this kind of solitary waves were also called classical solitary waves [8]. With the development of solitary waves theory, people realized that in addition to the classical solitary waves, there were other types of solitary waves. Hiroaki Ono considered the solitary waves in stratified fluids and found that in the case of great of depth, the behavior of long nonlinear waves was governed by an integrodifferential equation of dispersive type (BO equation) instead of the KdV equation [9]. This kind of solitary waves was called algebraic solitary waves. After the KdV theory and BO theory, a more general evolution equation for solitary waves in a finite-depth fluid, which reduces to the $\mathrm{KdV}$ equation in the shallow-fluid limit and to the BO equation in the infinitely-deep-fluid limit, was given by Kubota et al. [10]. The equation was called intermediate long waves (ILW) equation. Based on the above researches, many people carried on the research of solitary waves and explained some phenomena that occur in ocean and atmosphere, such as blocking and dipole [11-15].

As everyone knows ocean and atmosphere are driven by external forcing. The motion of ocean and atmosphere must be taken as a forced nonlinear system. Here the forcing factors include topographic forcing and external source forcing and so on. There have been lots of researches on the effect of topographic forcing on Rossby waves [12-18]. On the other hand, the real oceanic and atmospheric motion is dissipative, otherwise the motion would grow explosively because of the constant injecting of the external forcing energy. In particular, when the long-time evolution is considered, the small dissipation effects can become of crucial importance. They can stop self-similar expansion of the shock so that it tends to some stationary wave structure which propagates as a whole with constant velocity. But in many researches dissipation effect is ignored.

In the present paper, we will study the Rossby solitary waves generated by topography in finite depth fluids, especially we will consider the dissipation effect. Here we need emphasis that it seems very few ones on Rossby solitary waves excited by topography with a small dissipation in finite deep fluids by employing the forced ILW-Burgers equation are available up to now. This paper is organized as follows: in Section 2, a forced ILW-Burgers equation will be derived by using a perturbation method from the geostrophic potential vorticity equation with dissipation and topography effect. It will reduce to the KdV-Burgers equation in shallow fluids and to the BO-Burgers equation when the depth $h \rightarrow \infty$. So it is easy to find that the ILW-Burgers equation is an extension of the former researches. This is followed in Section 3 by the generation of the perturbation solution of the ILWBurgers equation and the discussion of dissipation effect. Section 4 is devoted to a study of the conservation relations associated with the equation and the conservation quantities of Rossby solitary waves. The numerical solutions of the forced ILW-Burgers equation are given for a topographic forcing by using the pseudospectral method in Section 5 . The solitary waves generated by topography are simulated, the effect of detuning parameter is analyzed. Especially, we carry out the comparison of Rossby solitary waves in finite deep fluids with that in infinite deep fluids. Finally, some conclusions are given in Section 6. 


\section{Mathematics Model}

The theoretical basis is found in the paper of Pedlosky [19] treating a model on a beta plane. In this model the vorticity equation governing the inviscid, quasi-geostrophic fluid motion with topography and turbulent dissipation, in the nondimensional form, is given by

$$
\left(\frac{\partial}{\partial t}+\frac{\partial \Psi}{\partial x} \frac{\partial}{\partial y}-\frac{\partial \Psi}{\partial y} \frac{\partial}{\partial x}\right)\left(\nabla^{2} \Psi+\beta y+h\right)=-\lambda_{0} \nabla^{2} \Psi+Q
$$

where $\Psi$ is the dimensionless stream function; $\nabla^{2}=\partial^{2} / \partial x^{2}+\partial^{2} / \partial y^{2}$ denotes the twodimensional Laplace operator; $\beta=\beta_{0}\left(L^{2} / U\right), \beta_{0}$ is the northward gradient of the planetary vorticity, $L$ and $U$ are the characteristic horizontal length and velocity scales; $\nabla^{2} \Psi$ expresses the vorticity dissipation which is caused by the Ekman boundary layer; $\lambda_{0}$ is a dissipative coefficient and $0 \leq \lambda_{0} \ll 1 ; Q$ is the external source, which is taken to be a function of $y$.

To restrict our consideration to a near-resonant system, we assume

$$
\Psi=-\int^{y}(U(s)-c+\varepsilon \alpha) d s+\varepsilon \psi,
$$

where $\alpha$ is a small disturbance in the basic flow and reflects the proximity of the system to a resonate state; $c$ is a constant, which is regarded as a Rossby waves phase speed; $\psi$ denotes disturbance stream-function. The substitution of (2.2) into (2.1) yields

$$
\left[\frac{\partial}{\partial t}+(U-c+\varepsilon \alpha) \frac{\partial}{\partial x}\right] \nabla^{2} \psi+\left(\beta-U^{\prime \prime}\right) \frac{\partial \psi}{\partial x}+\varepsilon J\left(\psi, \nabla^{2} \psi\right)+J(\psi, h)=-\lambda_{0} \nabla^{2} \psi
$$

Here, we divide the region into two parts: the domain $\left[0, h_{0}\right]$ and the domain $\left[h_{0}, h_{1}\right]$. In the domain $\left[0, h_{0}\right]$, in order to consider the role of nonlinearity, we must assume that shear of zonal flow exists, that is $U=u(y)$; while in the domain $\left[h_{0}, h_{1}\right]$, the parameter $\beta$ is smaller than that in the domain $\left[0, h_{0}\right]$, we can neglect constant $\beta$ in the domain $\left[h_{0}, h_{1}\right]$, meanwhile the zonal flow is assumed to be uniform, that is $U=u_{1}$, here $u_{1}$ is constant. Furthermore, in the domain $\left[h_{0}, h_{1}\right]$ the topography and turbulent dissipation is absent and only consider the features of disturbances generated. For simplicity, $u(y)$ is assumed to be smooth across $y=h_{0}$.

In the domain $\left[0, h_{0}\right]$, in order to achieve a balance among topography effect, turbulent dissipation and nonlinearity, we take

$$
h(x, y)=\varepsilon^{2} H(x, y), \quad \lambda_{0}=\varepsilon^{2} \lambda
$$

Substituting (2.4) into (2.3), yields

$$
\begin{aligned}
{\left[\frac{\partial}{\partial t}+\right.} & \left.(u-c+\varepsilon \alpha) \frac{\partial}{\partial x}\right] \nabla^{2} \psi^{(1)}+\left(\beta-u^{\prime \prime}\right) \frac{\partial \psi^{(1)}}{\partial x}+\varepsilon J\left(\psi^{(1)}, \nabla^{2} \psi^{(1)}+\varepsilon H\right) \\
& =-\varepsilon^{2} \lambda \nabla^{2} \psi^{(1)}, \quad y \in\left[0, h_{0}\right] .
\end{aligned}
$$


In the domain $\left[h_{0}, h_{1}\right]$, the governing equations is

$$
\left[\frac{\partial}{\partial t}+\left(u_{1}-c+\varepsilon \alpha\right) \frac{\partial}{\partial x}\right] \nabla^{2} \psi^{(2)}+\varepsilon J\left(\psi^{(2)}, \nabla^{2} \psi^{(2)}\right)=0, \quad y \in\left[h_{0}, h_{1}\right]
$$

The boundary conditions are

$$
\psi^{(1)} / y=0=\psi^{(2)} / y=h_{1}=0, \quad \psi^{(1)} / y=h_{0}=\psi^{(2)} / y=h_{0}, \quad \frac{\partial^{2} \psi^{(1)}}{\partial x \partial y} / y=h_{0}=\frac{\partial^{2} \psi^{(2)}}{\partial x \partial y} / y=h_{0},
$$

where $\psi^{(1)}$ denotes the disturbance streamfunction in the domain $\left[0, h_{0}\right], \psi^{(2)}$ is that in the domain $\left[h_{0}, h_{1}\right]$.

Following, let us consider the asymptotic expansions of (2.5) and (2.6). We introduce the following stretching transformation and the perturbation expansion in (2.5):

$$
\begin{gathered}
X=\varepsilon x, \quad T=\varepsilon^{2} t \\
\psi^{(1)}=\psi_{1}(X, y, T)+\varepsilon \psi_{2}(X, y, T)+\cdots .
\end{gathered}
$$

Substituting (2.8) into (2.5) leads to the following perturbation equations:

$$
\begin{gathered}
\varepsilon^{1}: \frac{\partial}{\partial X}\left(\frac{\partial^{2} \psi_{1}}{\partial y^{2}}+\frac{\beta-u^{\prime \prime}}{u-c} \psi_{1}\right)=0 \\
\varepsilon^{2}: \frac{\partial}{\partial X}\left(\frac{\partial^{2} \psi_{2}}{\partial y^{2}}+\frac{\beta-u^{\prime \prime}}{u-c} \psi_{2}\right)=\frac{-1}{u-c}\left[\left(\frac{\partial}{\partial T}+\alpha \frac{\partial}{\partial X}+\lambda\right) \frac{\partial^{2} \psi_{1}}{\partial y^{2}}-\frac{\partial \psi_{1}}{\partial y} \frac{\partial^{3} \psi_{1}}{\partial y^{2} \partial X}\right]-\frac{\partial H}{\partial X} .
\end{gathered}
$$

For the linear solution to be separable, we take the solution of (2.9) in the form

$$
\psi_{1}=A(X, T) \phi(y)
$$

then from (2.9) we can obtain

$$
\left(\frac{d^{2}}{d y^{2}}+\frac{\beta-u^{\prime \prime}}{u-c}\right) \phi(y)=0
$$

The Equation (2.12) is an eigenvalue problem and describes the space structure of the wave along direction. The boundary conditions can be obtained from (2.6). Equation (2.12) will be solved in the latter section.

For (2.10), we have

$$
\frac{\partial}{\partial X}\left(\frac{\partial^{2} \psi_{2}}{\partial y^{2}}+\frac{\beta-u^{\prime \prime}}{u-c} \psi_{2}\right)=\frac{1}{u-c}\left[\left(\frac{\partial A}{\partial T}+\alpha \frac{\partial A}{\partial X}+\lambda A\right) \frac{\beta-u^{\prime \prime}}{u-c} \phi+\left(\frac{\beta-u^{\prime \prime}}{u-c}\right)^{\prime} \phi^{2} A \frac{\partial A}{\partial X}\right]-\frac{\partial H}{\partial X}
$$


Multiplying the both sides of (2.13) by $\phi$ and integrating it with respect to $y$ from 0 to $h_{0}$, utilizing the boundary conditions (2.7), we get

$$
\begin{aligned}
\frac{\partial}{\partial X}\left[\phi \frac{\partial \psi_{2}}{\partial y}-\frac{d \phi}{d y} \psi_{2}\right]_{h_{0}}= & \left(\frac{\partial A}{\partial T}+\alpha \frac{\partial A}{\partial X}+\lambda A\right) \int_{0}^{h_{0}} \frac{\beta-u^{\prime \prime}}{(u-c)^{2}} \phi^{2} d y-\frac{\partial^{3} A}{\partial X^{3}} \int_{0}^{h_{0}} \phi^{2} d y \\
& +A \frac{\partial A}{\partial X} \int_{0}^{h_{0}}\left(\frac{\beta-u^{\prime \prime}}{u-c}\right)^{\prime} \phi^{3} d y-\int_{0}^{h_{0}} \phi \frac{\partial H}{\partial X} d y .
\end{aligned}
$$

The left hand of (2.14) can be determined by employing (2.6).

In the domain $\left[h_{0}, h_{1}\right]$, we adopt the transformations in the following forms:

$$
\xi=x, \quad T=\varepsilon^{2} t,
$$

and the disturbance streamfunction $\psi^{(2)}$ may be expressed by

$$
\psi^{(2)}=\tilde{\psi}(\xi, y, T, \varepsilon)
$$

Introducing (2.15) and (2.16) into (2.6), we can get the lowest-order equation for the domain $\left[h_{0}, h_{1}\right]$ as follows:

$$
\left(u_{1}-c\right) \frac{\partial}{\partial \xi}\left(\frac{\partial^{2}}{\partial \xi^{2}}+\frac{\partial^{2}}{\partial y^{2}}\right) \tilde{\psi}=0
$$

When taking the integration constant zero, we have

$$
\left(\frac{\partial^{2}}{\partial \xi^{2}}+\frac{\partial^{2}}{\partial y^{2}}\right) \tilde{\psi}=0
$$

According to Zhou [20], by virtue of the boundary condition $\tilde{\psi} / y=h_{1}=0$, we can obtain the solution of (2.18) as following:

$$
\widetilde{\psi}(\xi, y, T)=\frac{1}{2 \pi} \int_{-\infty}^{+\infty} F\left(\tilde{\psi} / y=h_{0}\right) \frac{\sinh p\left(h_{1}-y\right)}{\sinh p\left(h_{1}-h_{0}\right)} e^{i p \xi} d p
$$

where $F\left(\tilde{\psi} / y=h_{0}\right)$ denotes the Fourier transformation of $\tilde{\psi} / y=h_{0}$. Differentiating (2.19) with respect to $y$, we can obtain

$$
\frac{\partial \tilde{\psi}(\xi, y, T)}{\partial y}=\frac{-1}{2 \pi} \int_{-\infty}^{+\infty} F\left(\tilde{\psi} / y=h_{0}\right) p \frac{\cosh p\left(h_{1}-y\right)}{\sinh p\left(h_{1}-h_{0}\right)} e^{i p \xi} d p
$$


Assuming that the solution matches smoothly at $y=h_{0}$, then from (2.19) and (2.20), we get

$$
\begin{gathered}
\psi_{1}\left(X, h_{0}, T\right)+\varepsilon \psi_{2}\left(X, h_{0}, T\right)=\tilde{\psi}\left(\xi, h_{0}, T\right)+O\left(\varepsilon^{2}\right) \\
\frac{\partial \psi_{1}\left(X, h_{0}, T\right)}{\partial y}+\varepsilon \frac{\partial \psi_{2}\left(X, h_{0}, T\right)}{\partial y}=\frac{\partial \tilde{\psi}\left(\xi, h_{0}, T\right)}{\partial y}+O\left(\varepsilon^{2}\right)
\end{gathered}
$$

From (2.21), it is easy to find that

$$
A(X, T) \phi\left(y_{0}\right)=\tilde{\psi}\left(\xi, h_{0}, T\right), \quad \psi_{2}\left(X, h_{0}, T\right)=0
$$

Based on (2.20) and (2.23), we get

$$
\frac{\partial \tilde{\psi}\left(\xi, y_{0}, T\right)}{\partial y}=-\frac{\varepsilon}{R_{0}} \frac{\partial}{\partial X} \int_{-\infty}^{\infty} A\left(X^{\prime}, T\right) \phi\left(y_{0}\right) \operatorname{coth} \frac{\pi}{R_{0}}\left(X-X^{\prime}\right) d X^{\prime},
$$

where $R_{0}=2\left(h_{1}-h_{0}\right) \varepsilon$, and we take $h_{1}-h_{0}$ big enough to satisfy $R_{0} \geq O(1)$. So it is easy to obtain $\partial \tilde{\psi} / \partial y / y=h_{0}=O(\varepsilon)$. Then we obtain from (2.22) and (2.24)

$$
\phi^{\prime}\left(h_{0}\right)=0, \quad \frac{\partial \psi_{2}\left(X, h_{0}, T\right)}{\partial y}=-\frac{1}{R_{0}} \frac{\partial}{\partial X} \int_{-\infty}^{\infty} A\left(X^{\prime}, T\right) \phi\left(h_{0}\right) \operatorname{coth} \frac{\pi}{R_{0}}\left(X-X^{\prime}\right) d X^{\prime}
$$

Substituting (2.23) and (2.25) into (2.14) leads to

$$
\frac{\partial A}{\partial T}+\alpha \frac{\partial A}{\partial X}+a_{1} A \frac{\partial A}{\partial X}+a_{2} \frac{\partial^{2}}{\partial X^{2}} \int_{-\infty}^{\infty} A\left(X^{\prime}, T\right) \operatorname{coth} \frac{\pi}{R_{0}}\left(X-X^{\prime}\right) d X^{\prime}+\lambda A=a_{3} \frac{\partial H}{\partial X}
$$

where $a=\int_{0}^{h_{0}} \phi^{2}\left(\beta-u^{\prime \prime}\right) /(u-c) d y, a_{1}=\int_{0}^{h_{0}} \phi^{3}\left[\left(\beta-u^{\prime \prime}\right) /(u-c)\right]^{\prime} d y / a, a_{2}=\phi^{2}\left(h_{0}\right) /\left(R_{0} a\right)$, $a_{3}=\int_{0}^{h_{0}} \phi d y / a$. Equation (2.26) is a forced integrodifferential equation including dissipation and topography terms. In the absence of the topographic forcing and dissipation effect, (2.26) becomes the ILW equation. Here the term $\lambda A$ denotes the dissipation effect and has the same physical meaning with the term $\partial^{2} A / \partial X^{2}$ in Burgers equation, so we call (2.26) forced ILWBurgers equation. As we know that the so-called ILW-Burgers equation is first obtained here. In the absence of forcing and dissipation, (2.26) becomes the normal ILW equation

$$
\frac{\partial A}{\partial T}+\alpha \frac{\partial A}{\partial X}+a_{1} A \frac{\partial A}{\partial X}+a_{2} \frac{\partial^{2}}{\partial X^{2}} \int_{-\infty}^{\infty} A\left(X^{\prime}, T\right) \operatorname{coth} \frac{\pi}{R_{0}}\left(X-X^{\prime}\right) d X^{\prime}=0
$$

The solitary wave solution of (2.27) is

$$
A(X, T)=\frac{A_{0} \sin ^{2} \gamma}{\cosh ^{2} \tau_{1}(X-V T)-\cos ^{2} \gamma}
$$


where $A_{0}=-4 R_{0} a_{2} \gamma \cot \gamma / \pi a_{1}, \tau_{1}=-R_{0} \gamma / \pi, V=\alpha-\left(2 a_{2} R_{0} \gamma \cot 2 \gamma / \pi\right)$ is the propagation speed of the solitary waves, $\gamma$ is an amplitude parameter.

Here it should be noted that in some atmospheric and oceanic applications, the depth $h_{1} \rightarrow \infty, \operatorname{coth}\left(1 / R_{0}\right)\left(X-X^{\prime}\right) \rightarrow 1 /\left(X-X^{\prime}\right),(2.26)$ reduces to the BO-Burgers equation; in the opposite limit, (2.26) reduces to the KdV-Burgers equation. So we can conclude that the conditions describe by the KdV-Burgers equation and the BO-Burgers equation are special cases in the paper.

\section{Perturbation Solution of ILW-Burgers Equation}

In this section, in order to study the evolutional characters of Rossby solitary waves under the influence of dissipation, we need to seek for the solution of ILW-Burgers equation and the topography effect will be studied in the latter section. In the absence of the topographic forcing, (2.26) is reduced to the ILW-Burgers equation

$$
\frac{\partial A}{\partial T}+\alpha \frac{\partial A}{\partial X}+a_{1} A \frac{\partial A}{\partial X}+a_{2} \frac{\partial^{2}}{\partial X^{2}} \mathcal{L}(A(X, T))+\lambda A=0,
$$

where

$$
\mathcal{L}(A)=\int_{-\infty}^{\infty} A\left(X^{\prime}, T\right) \operatorname{coth} \frac{\pi}{R_{0}}\left(X-X^{\prime}\right) d X^{\prime}
$$

Next we study the perturbation solution of (3.1). Assume $\lambda \ll 1, \lambda \ll a_{1} \sim a_{2}$, let us take a new space coordinate

$$
\rho=X-\int_{0}^{T} \alpha-\frac{2 a_{2} R_{0} \gamma \cot 2 \gamma}{\pi} d T
$$

Assuming $A_{0}=A_{0}(\lambda T)$, then we have

$$
\frac{\partial A}{\partial T}+\frac{2 a_{2} R_{0} \gamma \cot 2 \gamma}{\pi} \frac{\partial A}{\partial \rho}+a_{1} A \frac{\partial A}{\partial \rho}+a_{2} \frac{\partial^{2} \mathcal{L}(A)}{\partial \rho^{2}}+\lambda A=0
$$

Taking two time scales

$$
\tau=T, \quad \eta=\lambda T,
$$

and expanding the solution as follows:

$$
A(\rho, T)=A_{1}(\rho, \tau, \eta)+\lambda A_{2}(\rho, \tau)+\cdots,
$$


we can obtain the following approximate equations:

$$
\begin{gathered}
\lambda^{0}: \frac{\partial A_{1}}{\partial \tau}+\frac{2 a_{2} R_{0} \gamma \cot 2 \gamma}{\pi} \frac{\partial A_{1}}{\partial \rho}+a_{1} A_{1} \frac{\partial A_{1}}{\partial \rho}+a_{2} \frac{\partial^{2} \mathcal{L}\left(A_{1}\right)}{\partial \rho^{2}}=0 \\
\lambda^{1}: \frac{\partial A_{2}}{\partial \tau}+\frac{2 a_{2} R_{0} \gamma \cot 2 \gamma}{\pi} \frac{\partial A_{2}}{\partial \rho}+a_{1} A_{1} \frac{\partial A_{2}}{\partial \rho}+a_{1} A_{2} \frac{\partial A_{1}}{\partial \rho}+a_{2} \frac{\partial^{2} \mathcal{L}\left(A_{2}\right)}{\partial \rho^{2}}=-\frac{\partial A_{1}}{\partial \eta}-A_{1} .
\end{gathered}
$$

Putting $\zeta=\rho-\left(2 a_{2} R_{0} \gamma \cot 2 \gamma / \pi\right) \tau$ into (3.7) gives

$$
\begin{gathered}
\lambda^{0}: \frac{\partial A_{1}}{\partial \tau}+a_{1} A_{1} \frac{\partial A_{1}}{\partial \zeta}+a_{2} \frac{\partial^{2} \mathcal{L}\left(A_{1}\right)}{\partial \zeta^{2}}=0 \\
\lambda^{1}: \frac{\partial A_{2}}{\partial \tau}+a_{1} A_{1} \frac{\partial A_{2}}{\partial \zeta}+a_{1} A_{2} \frac{\partial A_{1}}{\partial \zeta}+a_{2} \frac{\partial^{2} \mathcal{L}\left(A_{2}\right)}{\partial \zeta^{2}}=-\frac{\partial A_{1}}{\partial \eta}-A_{1} .
\end{gathered}
$$

Equation (3.8) is a normal ILW equation, based on (2.28), its solution is

$$
A_{1}(\zeta, \tau)=\frac{A_{0}(\lambda T) \sin ^{2} \gamma}{\cosh ^{2} \tau_{1}(\zeta-V \tau)-\cos ^{2} \gamma}
$$

where $A_{0}(\lambda T)=f(\gamma)=-4 R_{0} a_{2} \gamma \cot \gamma / \pi a_{1}$. So we can obtain the perturbation solution of the ILW-Burgers equation (3.1) as follows:

$$
A(X, T)=\frac{A_{0}(\lambda T) \sin ^{2} \gamma}{\cosh ^{2} \tau_{1}\left(X-\int_{0}^{T} \alpha-\left(2 a_{2} R_{0} \gamma \cot 2 \gamma / \pi\right) d T\right)-\cos ^{2} \gamma} .
$$
Assuming

In order to seek the expression of $A_{0}(\lambda T)$, we must consider the $\lambda^{1}$ equation (3.9).

$$
A_{2}=B(Y), \quad Y=\zeta-V \tau,
$$

from (3.9), we get

$$
-V \frac{d B}{d Y}+a_{1} \frac{\partial}{\partial Y}\left(A_{1} B+\frac{a_{2}}{a_{1}} \mathcal{L}\left(\frac{\partial B}{\partial Y}\right)\right)=M\left(A_{1}\right)
$$

where $M\left(A_{1}\right)=-\partial A_{1} / \partial \eta-A_{1}$. Equation (3.13) can be solved only when it satisfies

$$
\int_{-\infty}^{+\infty} G(Y) M\left(A_{1}\right) d Y=0
$$


Here $G(Y)$ satisfies the equation

$$
-V \frac{d G}{d Y}+a_{1} A_{1} \frac{d G}{d Y}-a_{2} \perp\left(\frac{d^{2} G}{d Y^{2}}\right)=0
$$

It is easy to find that in the case of $G( \pm \infty)=0$, the solution of (3.15) is $G(Y)=A_{0} \sin ^{2} \gamma$ $/\left(\cosh ^{2} \tau_{1} Y-\cos ^{2} \gamma\right)$. By employing the expression of $G(Y)$ and (3.14), we obtain

$$
A_{0}=\overline{A_{0}} e^{-\lambda T},
$$

where $\overline{A_{0}}$ is initial amplitude. So the perturbation solution of ILW-Burgers equation is

$$
A(X, T)=\frac{\overline{A_{0}} e^{-\lambda T} \sin ^{2} \gamma}{\cosh ^{2} \tau_{1}\left(X-\int_{0}^{T} \alpha-\left(2 a_{2} R_{0} \gamma \cot 2 \gamma / \pi\right) d T\right)-\cos ^{2} \gamma},
$$

where

$$
\gamma=f^{-1}\left(\overline{A_{0}} e^{-\lambda T}\right), \quad \tau_{1}=-\frac{R_{0} \gamma}{\pi}
$$

From Figures 1 and 2, it is obvious to find that the effect of a small dissipation is to cause the amplitude and moving speed of the solitary waves to decrease slowly with time.

In this section, we get the perturbation solution of ILW-Burgers equation and analyze the effect of dissipation on the amplitude and moving speed of solitary waves. While there are many methods to solve the nonlinear partial differential equation, both integrable and nonintegrable equation. In [21], a kind of new solutions of KdV equation called complexitons is presented; in $[22,23]$, the multiple expfunction method is successfully applied to many nonlinear partial differential equation, the rational function combinations of exponential functions will present good approximates to exact solutions; in [24, 25], a linear superposition principle of exponential traveling waves is analyzed for Hirota bilinear equations, with an aim to construct a specific subclass of N-soliton solutions formed by linear combinations of exponential traveling waves. We wonder if the above-mentioned methods can be applied to obtain the exact solution of ILW-Burgers equation. We will study these problems in the future.

\section{Conservation Laws}

Conservation laws are a common feature of mathematical physics, where they describe the conservation of fundamental physical quantities. The role played in the science by linear and nonlinear evolution equations, in particular, by conservation laws thereof, is hard to overestimate. It is well known that the $\mathrm{KdV}$ equation has an infinite number of conserved quantities, some multicomponent Burgers type equations, which possess infinitely many symmetries but not infinitely many conservation laws [26]. While four conservation quantities of BO equation are found by Ono in [9]. Next we will derive the conservation relations associated with (3.1). 


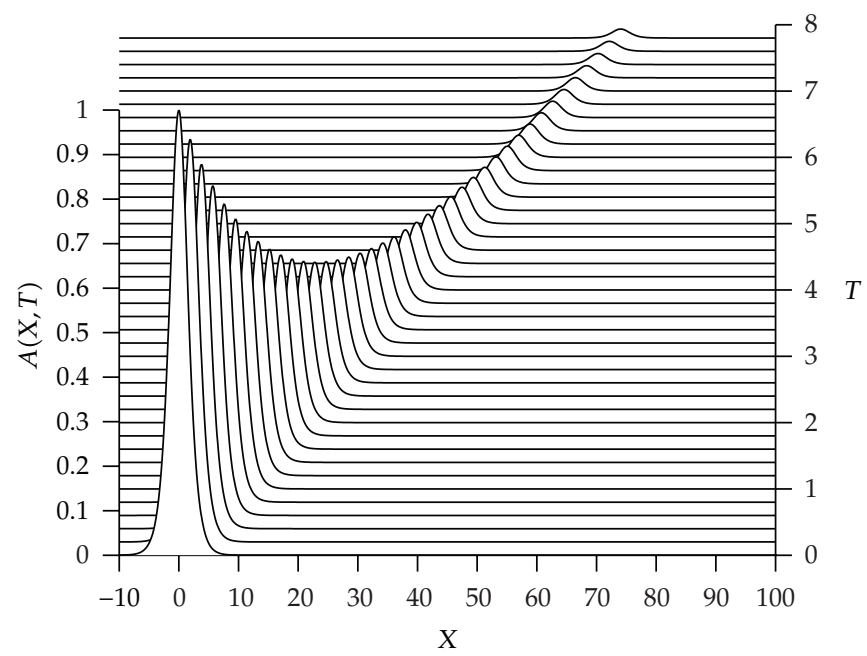

Figure 1: Perturbation solution of ILW-Burgers equation.

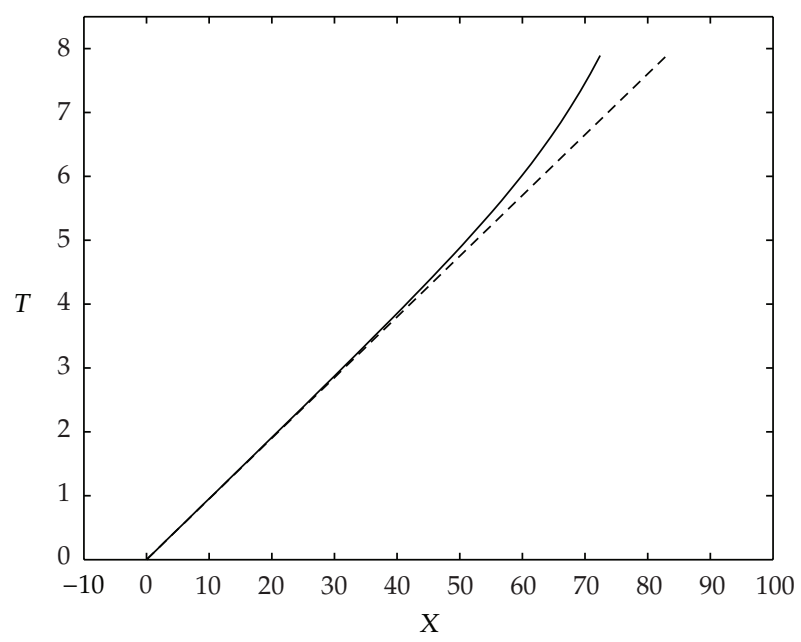

Figure 2: The trace of the vertex of solitary waves.

Following the general procedure of Ono [9], we assume that $A, A_{X}, A_{X X}$ vanish as $|X| \rightarrow \infty$ and integrating (3.1) and (3.1) by multiplying with powers of $A(X, T)$, we find the following two conservation relations:

$$
\begin{gathered}
E_{1}=\int_{-\infty}^{+\infty} A d X=\exp (-\lambda T) \int_{-\infty}^{+\infty} A(X, 0) d X \\
E_{2}=\int_{-\infty}^{+\infty} A^{2} d X=\exp (-2 \lambda T) \int_{-\infty}^{+\infty} A^{2}(X, 0) d X,
\end{gathered}
$$

where we employ the property of operator $\mathcal{L}: \int_{-\infty}^{+\infty} f(X) \mathcal{L}(g(X)) d X=$ $-\int_{-\infty}^{+\infty} g(X) \mathcal{L}(f(X)) d X$. Equation (4.1) show that $E_{1}$ and $E_{2}$ are two time-invariant quantities 
as the dissipation effect is neglected, that is $\lambda=0$. By analogy with the $\mathrm{KdV}$ equation, $E_{1}$ and $E_{2}$ are regarded as the mass and momentum of the solitary waves, respectively. So we conclude that the mass and momentum of the solitary waves are conserved without dissipation. Meanwhile, we can also get that the mass and momentum of the waves decrease exponentially with the increasing of time $T$ and the dissipative coefficient $\lambda$ in the presence of dissipation effect. Furthermore, the rate of decline of momentum is faster than the rate of mass.

Assume $\lambda=0$ and construct

$$
E_{3}=\int_{-\infty}^{+\infty}\left[\frac{1}{3} A^{3}+\frac{a_{2}}{a_{1}} \frac{\partial A}{\partial X} \mathcal{L}(A)\right] d X
$$

by adding $\left(A^{2}-\left(a_{2} / a_{1}\right) \mathcal{L}\left(A_{X}\right)\right) \times$ Equation (3.1) to $(\partial / \partial X)$ Equation (3.1) $\times\left[A_{X}+\right.$ $\left.\left(a_{2} / a_{1}\right) \mathcal{L}(A)\right]$ and integrating it, by virtue of the relation $\partial^{2} \mathcal{L}(A) / \partial X^{2}=\mathcal{L}\left(\partial^{2} A / \partial X^{2}\right)$, after tedious calculation, we find that $d E_{3} / d T=0$. Here $E_{3}$ expresses the energy of the solitary waves. So we can conclude that the energy of the solitary waves is conserved without dissipation.

Defining a quantity related to the phase of the solitary waves

$$
\widetilde{E}_{4}=\frac{d}{d T} \int_{-\infty}^{+\infty} X A d X
$$

we can deduce $d \widetilde{E}_{4} / d T=0$. While here we are interested in the quantity $E_{4}=\widetilde{E}_{4} / E_{1}$, which expresses the velocity of the center of gravity for the ensemble of such waves according to [9]. Then by using $d E_{1} / d T=0$ and $d \widetilde{E}_{4} / d T=0$, we obtain $d E_{4} / d T=0$, which shows that the velocity of the center of gravity is conserved without dissipation.

In fact, beside the above four conservation relations, we can also verify the invariance of the following quantity:

$$
E_{5}=\int_{-\infty}^{+\infty}\left[\frac{1}{4} A^{4}+\frac{3 a_{2}}{2 a_{1}} A^{2} \frac{\partial}{\partial X} \mathcal{L}(A)+\frac{1}{18}\left(\frac{\partial A}{\partial X}\right)^{2}\right] d X
$$

In this section, we obtain five conservation relations of ILW-Burgers equation and draw the conclusion that the dissipation effect causes the mass, the momentum, the energy, and the velocity of the center of gravity to vary. In fact, after the above five conservation laws are given, we can wonder whether there exist other conservation laws? Is there an infinite number of conservation laws like the KdV equation? What deserves to be studied in the future?

\section{Numerical Simulation and Discussion}

In Section 4, we have obtained the conservation relations of the homogenous ILW-Burgers equation. In this section, we will take into account the influence of topography for the Rossby solitary waves. Rossby solitary waves excited by topography with dissipation will be discussed. In order to resolve the above problems, we need to solve the forced ILW-Burgers equation. There is no analytical solution for this equation, here we will present numerical solutions of (2.26) by using the pseudospectral method [27]. First, let us simply introduce the method. 
The pseudospectral method uses a Fourier transform treatment of the space dependence together with a leap-forg scheme in time. For ease of presentation the spatial period is normalized to $[0,2 \pi]$. This interval is divided into $2 N$ points, then $\Delta T=\pi / N$. The function $A(X, T)$ can be transformed to the Fourier space by

$$
\widehat{A}(v, T)=F A=\frac{1}{\sqrt{2 N}} \sum_{j=0}^{2 N-1} A(j \Delta X, T) e^{-\pi i j v / N}, \quad v=0, \pm 1, \ldots, \pm N
$$

The inversion formula is

$$
A(j \Delta X, T)=F^{-1} \widehat{A}=\frac{1}{\sqrt{2 N}} \sum_{v} \widehat{A}(v, T) e^{\pi i j v / N}
$$

These transformations can use fast Fourier Transform algorithm to efficiently perform. With this scheme, $\partial A / \partial X$ can be evaluated as $F^{-1}\{i v F A\}, \partial^{2} A / \partial X^{2}$ as $-F^{-1}\left\{v^{2} F A\right\}, \partial H / \partial X$ as $F^{-1}\{i v F H\}$ and so on. Combined with a leap-frog time step, (2.26) would be approximated by

$$
\begin{gathered}
A(X, T+\Delta T)-A(X, T-\Delta T)+i \alpha F^{-1}\{v F A\} \Delta T+i a_{1} A F^{-1}\{v F A\} \Delta T+\lambda A \\
-a_{2} \Delta T F^{-1}\left\{v^{2} F \mathcal{L}(A)\right\}=i a_{3} F^{-1}\{v F H\} \Delta T .
\end{gathered}
$$

The computational cost for (5.3) is six fast Fourier transforms per time step.

Before solving (2.26) by using the pseudospectral method, we need to obtain the coefficients $a_{1}, a_{2}$, and $a_{3}$.

Taking $\phi_{1} / y=h_{0}=-1$, combining (2.12) and the boundary conditions (2.7), we can obtain the following eigenvalue problem:

$$
\begin{aligned}
& \left(\frac{d^{2}}{d y^{2}}+\frac{\beta-u^{\prime \prime}}{u-c}\right) \phi(y)=0 \\
& \phi(0)=0, \quad \phi / y=h_{0}=-1 .
\end{aligned}
$$

Assume the weak shear flow to be $u=u_{0}+\delta y$, where $u_{0}$ is a constant, $\delta \leq 1$ shows that the flow shear is weak. We may get approximate expressions for $\phi$ and $c_{0}$ in powers of $\delta$ as follows:

$$
\begin{gathered}
\phi=\phi_{1}+\delta \phi_{2}+\cdots, \\
c=c_{1}+\delta c_{2}+\cdots .
\end{gathered}
$$


Substituting (5.5) into (5.4), we obtain

$$
\begin{gathered}
\delta^{0}:\left\{\begin{array}{l}
\left(\frac{d^{2}}{d y^{2}}+\frac{\beta-u^{\prime \prime}}{u-c_{1}}\right) \phi_{1}=0, \\
\phi_{1}(0)=0, \quad \phi_{1} / y=h_{0}=-1,
\end{array}\right. \\
\delta^{1}:\left\{\begin{array}{l}
\left(\frac{d^{2}}{d y^{2}}+\frac{\beta-u^{\prime \prime}}{u-c_{1}}\right) \phi_{2}=\frac{c_{2}-y}{u_{0}-c_{1}} \frac{d^{2} \phi_{1}}{d y^{2}} \\
\phi_{2}(0)=0, \quad \phi_{2} / y=h_{0}=0 .
\end{array}\right.
\end{gathered}
$$

The solutions of $\phi_{1}$ in (5.6)

$$
\begin{gathered}
\phi_{1}=-\sin m y, \\
c_{1}=u_{0}-\frac{\beta}{m^{2}}, \quad m=\left(2 n+\frac{1}{2}\right) \frac{\pi}{h_{0}}, \quad n=0, \pm 1, \pm 2, \ldots .
\end{gathered}
$$

Substituting them into (5.6), we have

$$
\begin{gathered}
\phi_{2}=-\frac{m^{2}}{4 \beta} y \sin m y+\frac{m^{3}}{4 \beta}\left(y^{2}-y\right) \cos m y, \\
c_{2}=\frac{1}{2}, \quad m=\left(2 n+\frac{1}{2}\right) \frac{\pi}{h_{0}}, \quad n=0, \pm 1, \pm 2, \ldots
\end{gathered}
$$

Taking approximately $\phi \simeq \phi_{1}+\delta \phi_{2}, c \simeq c_{1}+\delta c_{2}$. Employing (5.7) and (5.8), we can get the approximate solutions for $\phi$ and $c$.

As an example of calculation, we take $u=0.50-0.304 y$ and the topography profile is taken to be the Gaussian distribution as follows:

$$
H=H_{0} * \exp \left[-\frac{\left(X-X_{0}\right)^{2}+\left(y-y_{0}\right)^{2}}{d^{2}}\right]
$$

where $d=2, H_{0}=1, X_{0}=0, y_{0}=0$.

The time evolutions of the solitary waves generated by the topography in the absence of dissipation are shown in Figure 3 for different detuning parameter $\alpha$. From Figure 3 , it is easy to find that the detuning parameter $\alpha$ holds important implications for the evolution feature of the solitary waves. In the case of $\alpha>0$, the topographic forcing generates a steady solitary wave in the forcing region. The amplitude of solitary waves increases slightly with time at the beginning and then remains invariable. A modulated cnoidal wave-train with small amplitude is in the downstream region and there is no wave in the upstream region. Between the solitary wave and the modulated wave-train, there exists a buffer region. With the decreasing of $\alpha$, the modulated cnoidal wave-train approaches to the topographic forcing region. The buffer region between the solitary wave and modulated cnoidal wave-train disappears slowly. 


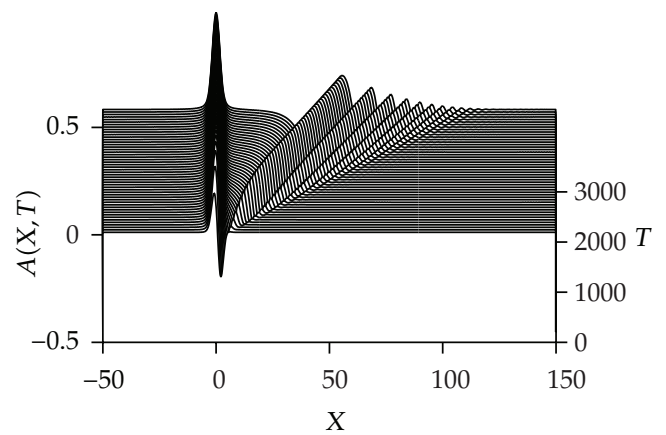

(a)

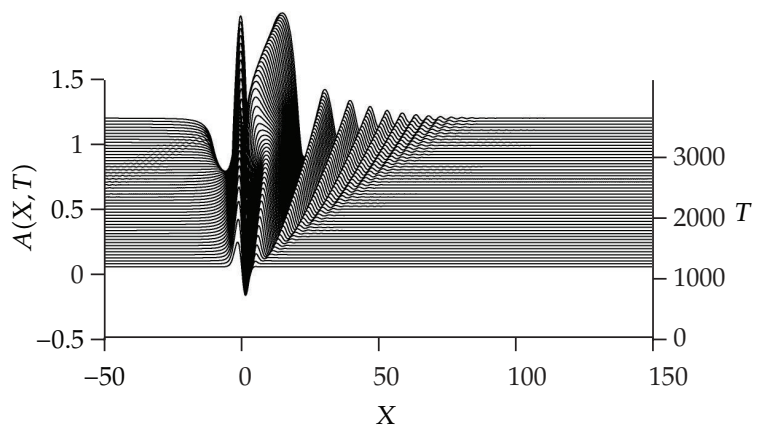

(b)

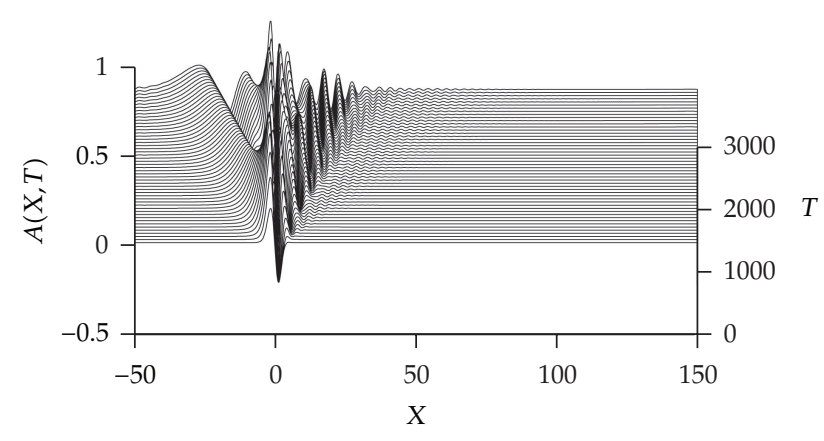

(c)

Figure 3: Solitary waves generated by topography in the absent of dissipation, (a) $\alpha=1.5 \times 10^{-2}$; (b) $\alpha=0$; (c) $\alpha=-1.5 \times 10^{-2}$.

When $\alpha=0$, the system gets to a resonant state (Figure 3(b)). In this case, a nonsteady solitary wave is formed in the forcing region whose amplitude is larger than that in Figure 3(a). There is also a modulated cnoidal wave-train in the downstream region and there is no wave in the upstream region. The amplitude of modulated cnoidal wave-train becomes larger and the wavelength becomes shorter.

As $\alpha$ decreases further and becomes to be negative value (Figure 3(c)), there generates a complex nonsteady wave near the forcing region. The amplitude of wave in the forcing region is larger than that in Figure 3(a) and smaller than that in Figure 3(b). The wavelength of the modulated cnoidal wave-train which exists in the downstream region is the smallest among the Figure 3. Similar with Figure 3(b), there is no buffer region between the wave in 


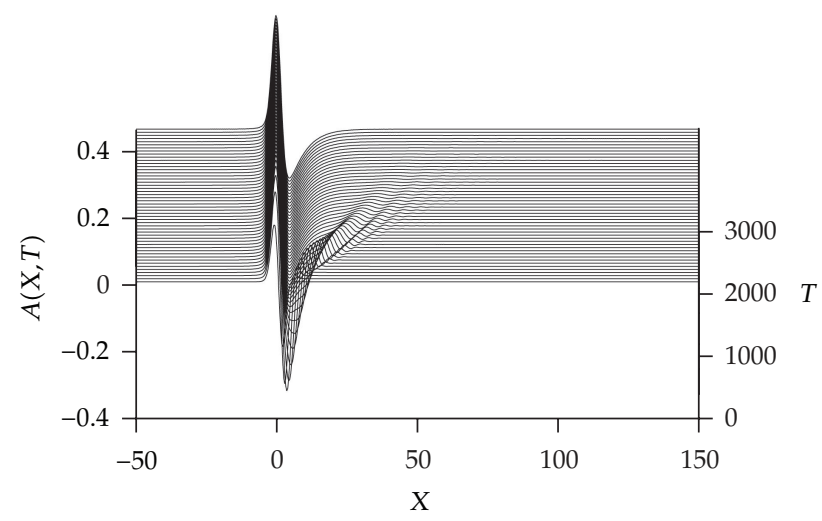

Figure 4: Solitary waves generated by topography in the presence of dissipation $\lambda=0.3 \times 10^{-2}, \alpha=1.5 \times 10^{-2}$.

the forcing and modulated cnoidal wave-train. Unlike the Figures 3(a) and 3(b), there is also modulated cnoidal wave-train in the upstream region.

In fact, by comparing the Figure 3 in this paper with the Figure 1 in [16], which the amplitude of solitary waves is governed by the BO-Burgers equation, we can find that the solitary waves governed by ILW-Burgers equation and BO-Burgers equation exist obvious difference. For the case $\alpha=0$ in [16], there is still a standing solitary wave in the forcing region, but it becomes a negative one, when $\alpha<0$, a negative standing solitary wave is formed in the forcing region, which is a stationary solitary wave disturbance. Based on the above discussion, we can conclude that the solitary waves generated by topography in finite depth fluids are different from that in infinite depth fluids.

Next we will discuss the solitary waves generated by topography in the presence of dissipation. Here we only consider the case that the detuning parameter $\alpha$ is positive, the other cases are omitted. Comparing Figure 4 with Figure 3(a), we can find that a solitary wave is also generated in the forcing region, but it is nonsteady, its amplitude decreases with time because of the dissipation effect. Meanwhile, the dissipation causes the modulated cnoidal wave-train in the downstream region to be dissipated.

\section{Summary}

In the paper, a new governing equation (forced ILW-Burgers) is derived to describe the amplitude of Rossby solitary waves generated by topography under the influence of dissipation. Neglecting the topography effect, the perturbation solution of ILW-Burgers is given. From the perturbation solution, we can find that the effect of a small dissipation is to cause the amplitude and moving speed of the solitary waves to decrease slowly with time. Following, five conserved quantities are obtained from the ILW equation. Based on these conserved quantities, we can conclude that the mass, momentum, energy, velocity of the center of gravity of Rossby solitary waves are conserved. Finally, the forced ILW-Burgers equation is solved numerically by using the pseudospectral method. When the dissipation is absent, the numerical results show that the detuning parameter have an important effect on the solitary waves generated by topography. The smaller the parameter $|\alpha|$ is, the amplitude of solitary wave in the forcing region is larger and the solitary wave is less steady. In addition, comparing with the solitary waves governed by ILW-Burgers equation and BO-Burgers equation, we can 
conclude that the solitary waves generated by topography in deep fluids are different from that in infinite depth fluids. By discussing the solitary waves generated by topography in the presence of dissipation, we can find that the dissipation causes the amplitude of the solitary wave in the forcing region to decrease and the modulated cnoidal wave-train to be dissipated.

\section{Acknowledgments}

This work was supported by National Natural Science Foundation of China (no. 40976008), Innovation Project of Chinese Academy of Sciences (no. KZCX2-EW-209), National Natural Science Foundation of China (no. 51174128), and Graduate Innovation Foundation from Shandong University of Science and Technology (no. YCA120212).

\section{References}

[1] A. C. Scott, F. Y. F. Chu, and D. W. McLaughlin, "The soliton: a new concept in applied science," Procedings of the IEEE, vol. 61, pp. 1443-1483, 1973.

[2] R. R. Long, "Solitary waves in the westerlies," Journal of the Atmospheric Sciences, vol. 21, pp. 197-200, 1964.

[3] D. J. Benney, "Long non-linear waves in fluid flows," Journal of Mathematical Physics, vol. 45, pp. 52-63, 1966.

[4] L. G. Redekopp, "On the theory of solitary Rossby waves," Journal of Fluid Mechanics, vol. 82, no. 4, pp. 725-745, 1977.

[5] R. Grimshaw, "Nonlinear aspects of long shelf waves," Geophysical and Astrophysical Fluid Dynamics, vol. 8, no. 1, pp. 3-16, 1977.

[6] J. P. Boyd, "Equatorial solitary waves. Part 1: rossby solitons," Journal of Physical Oceanography, vol. 10, no. 11, pp. 1699-1717, 1980.

[7] T. Yamagata, "On nonlinear planetary waves: a class of solutions missed by the traditional quasigeostrophic approximation," Journal of the Oceanographical Society of Japan, vol. 38, no. 4, pp. 236-244, 1982.

[8] J. I. Yano and Y. N. Tsujimura, "The domain of validity of the KdV-type solitary rossby waves in the shallow water $\beta$-plane model," Dynamics of Atmospheres and Oceans, vol. 11, no. 2, pp. 101-129, 1987.

[9] H. Ono, "Algebraic solitary waves in stratified fluids," Journal of the Oceanographical Society of Japan, vol. 39, no. 4, pp. 1082-1091, 1975.

[10] T. Kubota, D. R. S. Ko, and L. D. Dobbs, "Weakly-nonlinear, long internal gravity waves in stratified fluids of finite depth," Journal of Hydrology, vol. 12, no. 4, pp. 157-165, 1978.

[11] D. H. Luo, "Algebraic solitary Rossby wave in the atmosphere," Acta Meteorologica Sinica, vol. 49, pp. 269-277, 1991.

[12] G. Gottwald and R. Grimshaw, "The effect of topography on the dynamics of interacting solitary waves in the context of atmospheric blocking," Journal of the Atmospheric Sciences, vol. 56, no. 21, pp. 3663-3678, 1999.

[13] D. Luo, "A barotropic envelope Rossby soliton model for block-eddy interaction. I. Effect of topography," Journal of the Atmospheric Sciences, vol. 62, no. 1, pp. 5-21, 2005.

[14] O. E. Polukhina and A. A. Kurkin, "Improved theory of nonlinear topographic Rossby waves," Oceanology, vol. 45, no. 5, pp. 607-616, 2005.

[15] Z. X. Luo, N. E. Davidson, F. Ping, and W. C. Zhou, "Multiple-scale interactions affecting tropical cyclone track changes," Advances in Mechanical Engineering, vol. 2011, Article ID 782590, 9 pages, 2011.

[16] L. Meng and K. L. Lv, "Dissipation and algebraic solitary long-waves excited by localized topography," Chinese Journal of Computational Physics, vol. 19, pp. 159-167, 2002.

[17] L. Yang, C. Da, J. Song, H. Zhang, H. Yang, and Y. Hou, "Rossby waves with linear topography in barotropic fluids," Chinese Journal of Oceanology and Limnology, vol. 26, no. 3, pp. 334-338, 2008.

[18] H. W. Yang, B. S. Yin, D. Z. Yang, and Z. H. Xu, "Forced solitary Rossby waves under the influence of slowly varying topography with time," Chinese Physics B, vol. 20, no. 12, Article ID 120203, 2011.

[19] J. Pedlosky, Geophysical Fluid Dynamics, Springer, New York, NY, USA, 1979.

[20] Q. F. Zhou, "The second-order solitary waves in stratified fluids with finite depth," Scientia Sinica Series A, vol. 28, no. 2, pp. 159-171, 1985. 
[21] W.-X. Ma, "Complexiton solutions of the Korteweg-de Vries equation with self-consistent sources," Chaos, Solitons E Fractals, vol. 26, no. 5, pp. 1453-1458, 2005.

[22] W. X. Ma, T. Huang, and Y. Zhang, "A multiple exp-function method for nonlinear differential equations and its application," Physica Scripta, vol. 82, no. 6, Article ID 065003, 2010.

[23] W.-X. Ma and Z. Zhu, "Solving the $(3+1)$-dimensional generalized KP and BKP equations by the multiple exp-function algorithm," Applied Mathematics and Computation, vol. 218, no. 24, pp. 1187111879, 2012.

[24] W.-X. Ma and E. Fan, "Linear superposition principle applying to Hirota bilinear equations," Computers $\mathcal{E}$ Mathematics with Applications, vol. 61, no. 4, pp. 950-959, 2011.

[25] W.-X. Ma, Y. Zhang, Y. Tang, and J. Tu, "Hirota bilinear equations with linear subspaces of solutions," Applied Mathematics and Computation, vol. 218, no. 13, pp. 7174-7183, 2012.

[26] W.-X. Ma and Z.-X. Zhou, "Coupled integrable systems associated with a polynomial spectral problem and their Virasoro symmetry algebras," Progress of Theoretical Physics, vol. 96, no. 2, pp. 449 $457,1996$.

[27] B. Fornberg, A Practical Guide to Pseudospectral Methods, vol. 1, Cambridge University Press, Cambridge, UK, 1996. 


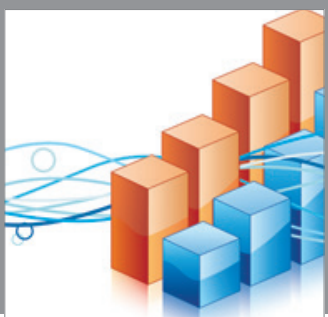

Advances in

Operations Research

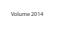

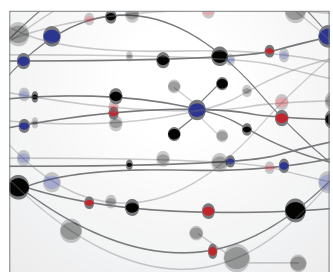

\section{The Scientific} World Journal
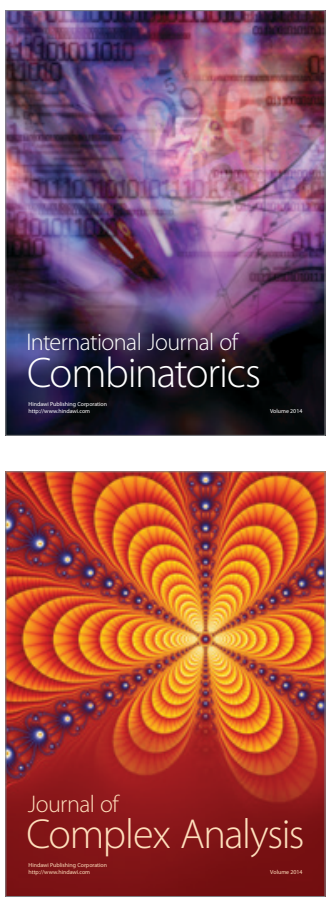

International Journal of

Mathematics and

Mathematical

Sciences
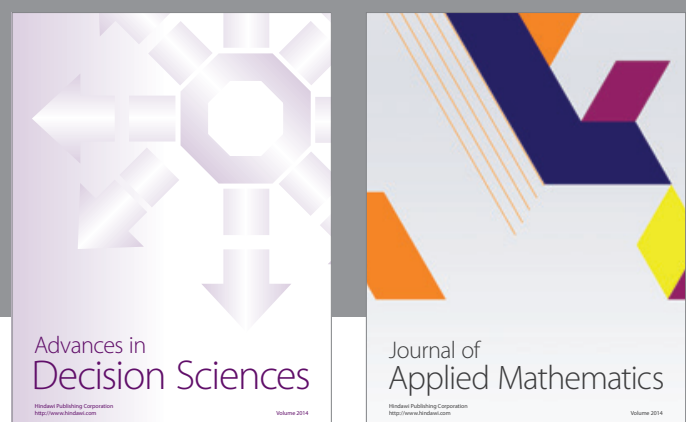

Journal of

Applied Mathematics
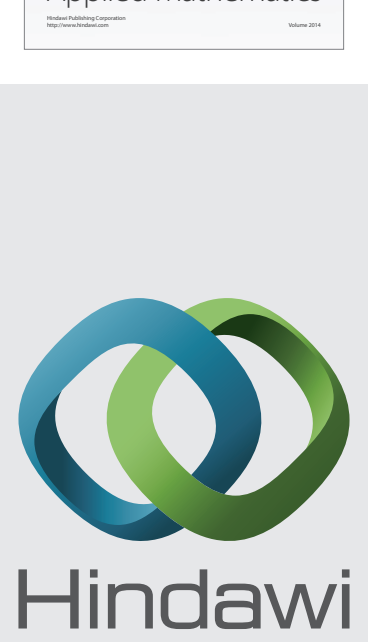

Submit your manuscripts at http://www.hindawi.com
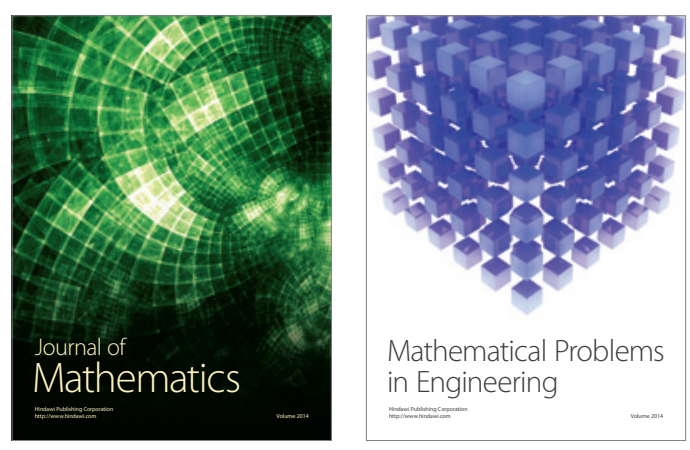

Mathematical Problems in Engineering
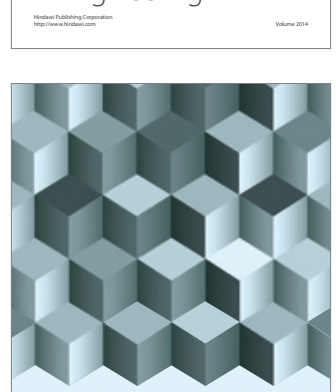

Journal of

Function Spaces
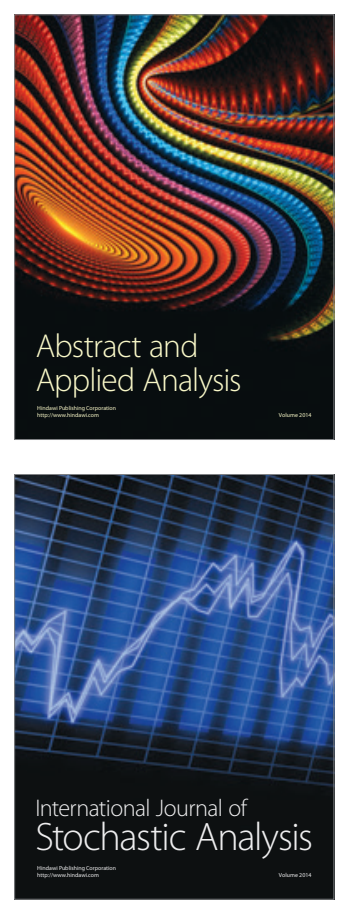

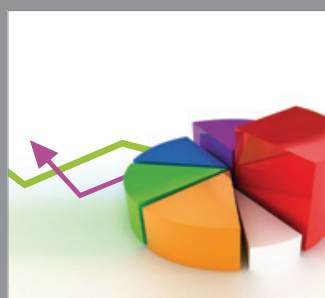

ournal of

Probability and Statistics

Promensencen
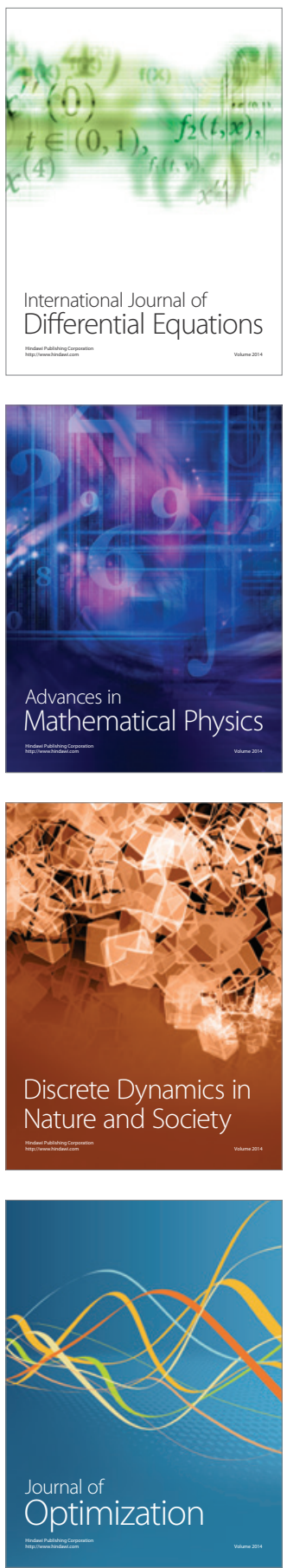